\title{
Unique binding behavior of the recently approved angiotensin II receptor blocker azilsartan compared with that of candesartan
}

\begin{abstract}
Shin-ichiro Miura ${ }^{1,2,3}$, Atsutoshi Okabe ${ }^{4}$, Yoshino Matsuo ${ }^{1}$, Sadashiva S Karnik ${ }^{3}$ and Keijiro Saku ${ }^{1,2}$
The angiotensin II type $1\left(\mathrm{AT}_{1}\right)$ receptor blocker (ARB) candesartan strongly reduces blood pressure (BP) in patients with hypertension and has been shown to have cardioprotective effects. A new ARB, azilsartan, was recently approved and has been shown to provide a more potent 24-h sustained antihypertensive effect than candesartan. However, the molecular interactions of azilsartan with the $\mathrm{AT}_{1}$ receptor that could explain its strong BP-lowering activity are not yet clear. To address this issue, we examined the binding affinities of ARBs for the $\mathrm{AT}_{1}$ receptor and their inverse agonist activity toward the production of inositol phosphate (IP), and we constructed docking models for the interactions between ARBs and the receptor. Azilsartan, unlike candesartan, has a unique moiety, a 5-oxo-1,2,4-oxadiazole, in place of a tetrazole ring. Although the results regarding the binding affinities of azilsartan and candesartan demonstrated that these ARBs interact with the same sites in the $\mathrm{AT}_{1}$ receptor ( $\mathrm{Tyr}^{113}$, Lys ${ }^{199}$ and $\mathrm{Gln}^{257}$ ), the hydrogen bonding between the oxadiazole of azilsartan-Gln ${ }^{257}$ is stronger than that between the tetrazole of candesartan-GIn 257 , according to molecular docking models. An examination of the inhibition of IP production by ARBs using constitutively active mutant receptors indicated that inverse agonist activity required azilsartanGIn 257 interaction and that azilsartan had a stronger interaction with $\mathrm{Gln}^{257}$ than candesartan. Thus, we speculate that azilsartan has a unique binding behavior to the $\mathrm{AT}_{1}$ receptor due to its 5-oxo-1,2,4-oxadiazole moiety and induces stronger inverse agonism. This property of azilsartan may underlie its previously demonstrated superior BP-lowering efficacy compared with candesartan and other ARBs.

Hypertension Research (2013) 36, 134-139; doi:10.1038/hr.2012.147; published online 4 October 2012
\end{abstract}

Keywords: azilsartan; candesartan; inverse agonist; oxadiazole

\section{INTRODUCTION}

The angiotensin II (Ang II) type $1\left(\mathrm{AT}_{1}\right)$ receptor, which is a member of the G-protein-coupled receptor superfamily, has a widespread tissue distribution and mediates most known cardiovascular functions. ${ }^{1} \mathrm{AT}_{1}$ receptor blockers (ARBs) are highly selective for the $\mathrm{AT}_{1}$ receptor and block the diverse effects of Ang II.

Azilsartan, a new ARB, was recently approved for the treatment of hypertension and is the eight ARB in clinical use worldwide. Azilsartan medoxomil, a prodrug of azilsartan, was approved in the United States by the Food and Drug Administration in 2011 for the treatment of hypertension. Azilsartan was approved for use in Japan in 2012. Azilsartan medoxomil and azilsartan have been shown to have greater antihypertensive effects than other ARBs. ${ }^{2-5}$ Treatment with azilsartan medoxomil lowered 24-h blood pressure (BP) significantly more than treatment with olmesartan medoxomil or valsartan. ${ }^{2,3}$ In addition, a multicenter, randomized, double-blind study compared the efficacy and safety of azilsartan with that of candesartan cilexetil in 622 Japanese patients with grade I-II essential hypertension. The results showed that azilsartan provided a more potent $24 \mathrm{~h}$ sustained antihypertensive effect than candesartan, but with equivalent safety. ${ }^{5}$ Azilsartan was discovered by modification of the tetrazole ring in candesartan, and has a unique moiety, a 5-oxo1,2,4-oxadiazole, in place of a tetrazole ring. ${ }^{6}$ Most ARBs have class (or common) effects because they have common molecular structures (biphenyl-tetrazole and imidazole groups; Supplementary Figure 1). ARBs have been shown to have class- and molecule-specific differential effects in basic experimental studies. ${ }^{7}$ We also proposed that small differences in the molecular structures of ARBs could lead to differences in their abilities to influence the $\mathrm{AT}_{1}$ receptor, ${ }^{7,8}$ as small differences in ligands for other G-protein-coupled receptors could lead to differences in pharmacological effects. ${ }^{9,10}$ In comparison with other ARBs, azilsartan bound tightly to and dissociated slowly from $\mathrm{AT}_{1}$ receptors. ${ }^{11}$ In addition, azilsartan induced the insurmountable antagonism of Ang II-induced vascular contractions against $\mathrm{AT}_{1}$ receptor.

${ }^{1}$ Department of Cardiology, Fukuoka University School of Medicine, Fukuoka, Japan; ${ }^{2}$ Department of Molecular Cardiovascular Therapeutics, Fukuoka University School of Medicine, Fukuoka, Japan; ${ }^{3}$ Department of Molecular Cardiology, Lerner Research Institute, The Cleveland Clinic Foundation, Cleveland, OH, USA and ${ }^{4}$ Pharmaceutical Research Division, Discovery Research Center, Takeda Pharmaceutical Company Limited, Kanagawa, Japan

Correspondence: Dr S Miura, Department of Cardiology, Fukuoka University School of Medicine, 7-45-1 Nanakuma, Jonan-ku, Fukuoka 814-0180, Japan.

E-mail: miuras@cis.fukuoka-u.ac.jp

Received 22 May 2012; revised 2 July 2012; accepted 7 July 2012; published online 4 October 2012 
We previously reported that the $\mathrm{AT}_{1}$ receptor exhibits a low level of constitutive activity in the absence of any ligand. ${ }^{12}$ Small differences in the chemical structures of ligands can be responsible for agonism, neutral antagonism or inverse agonism toward a G-protein-coupled receptor. ${ }^{13,14}$ Therefore, we hypothesized that the 5-oxo-1,2,4-oxadiazole moiety of azilsartan, which represents a small difference in the molecular structures of azilsartan and candesartan, may be responsible for the molecular effects of azilsartan, such as inverse agonism. We examined the binding affinities of azilsartan and candesartan to the $\mathrm{AT}_{1}$ receptor, along with their inverse agonist activity, and we constructed molecular docking models for the comparison of the interactions between these ARBs and the receptor.

\section{MATERIALS AND METHODS}

\section{Materials}

The following antibodies and reagents were purchased or provided: candesartan, azilsartan and azilsartan-7H, which does not contain a carboxyl group in the benzimidazole ring compared with azilsartan (Takeda Pharm, Osaka, Japan); $\left[\mathrm{Sar}^{1}\right]$ Ang II and $\left[\mathrm{Sar}^{1}, \mathrm{Ile}^{8}\right]$ Ang II (Sigma-Aldrich, St Louis, MO, USA); and ${ }^{125} \mathrm{I}_{-}\left[\mathrm{Sar}^{1}, \mathrm{Ile}^{8}\right]$ Ang II (Amersham Biosciences, Buckinghamshire, UK).

\section{Mutagenesis and expression of the $\mathrm{AT}_{1}$ receptor and membrane preparation}

The synthetic wild-type (WT) $\mathrm{AT}_{1}$ receptor gene, cloned in the shuttle expression vector pMT-3, was used for expression and mutagenesis studies, as described previously. ${ }^{13,15}$

\section{Cell cultures, transfections and membrane preparation}

COS1 cells were cultured in 10\% fetal bovine serum and penicillin- and streptomycin-supplemented Dulbecco's modified Eagle's essential medium (Invitrogen, Carlsbad, CA, USA) in $5 \% \mathrm{CO}_{2}$ at $37^{\circ} \mathrm{C}$. In the experiments, cells without cell-growth supplement were used. Cell viability in control experiments was $>95 \%$ by trypan blue exclusion analysis. The WT and mutant $\mathrm{AT}_{1}$ receptors were transiently transfected into COS1 cells using Lipofectamine 2000 liposomal reagent (Invitrogen) according to the manufacturer's instructions. Cell membranes were prepared by the nitrogen Parr bomb disruption method in the presence of protease inhibitors.

\section{Competition binding study}

The $K_{\mathrm{d}}$ values of receptor binding were determined by ${ }^{125} \mathrm{I}-\left[\mathrm{Sar}^{1}, \mathrm{Ile}^{8}\right]$ AngIIbinding experiments under equilibrium conditions, as described previously. ${ }^{13,15}$ Cell membranes expressing the WT or mutant receptor were incubated at room temperature for $1 \mathrm{~h}$ with ${ }^{125} \mathrm{I}-\left[\mathrm{Sar}^{1}, \mathrm{Ile}^{8}\right]$ Ang II. All binding experiments were carried out at $22^{\circ} \mathrm{C}$ in a volume of $125 \mu$ l. Nonspecific binding to the membranes was determined from ${ }^{125} \mathrm{I}-\left[\mathrm{Sar}^{1}, \mathrm{Ile}^{8}\right]$ Ang II binding in the presence of $10 \mu \mathrm{M}\left[\mathrm{Sar}^{1}, \mathrm{Ile}^{8}\right]$ Ang II. After equilibrium was reached, the binding experiments were stopped by filtering the binding mixture through Whatman GF/C glass fiber filters, which were extensively washed further with binding buffer to wash the free radioligand. The bound ligand fraction was determined from the c.p.m. remaining on the membrane. Equilibrium-binding kinetics were determined as described previously. ${ }^{13,15}$

\section{Inositol phosphate (IP) production assay}

Agonist- or ARB-induced IP formation by WT and mutant $\mathrm{AT}_{1}$ receptors in transfected cells was measured to evaluate cell signaling for vasoconstriction. Semi-confluent COS-1 cells transfected in $60 \mathrm{~mm}$ petri dishes were labeled for $24 \mathrm{~h}$ with $\left[{ }^{3} \mathrm{H}\right]$-myoinositol at $37^{\circ} \mathrm{C}$ in Dulbecco's Modified Eagle Medium containing $10 \%$ fetal bovine serum. On the day of the functional assay, the labeled cells were washed with Hank's balanced salt solution three times and incubated with Hank's balanced salt solution containing $10 \mathrm{~mm} \mathrm{LiCl}$ for $20 \mathrm{~min}$; $1 \mu \mathrm{M}$ ARBs or $0.1 \mu \mathrm{M}$ [ $\mathrm{Sar}^{1}$ ] Ang II was added and incubation was continued for another $10 \mathrm{~min}$ at $37^{\circ} \mathrm{C}$. At the end of incubation, the medium was removed, and total soluble IP was extracted from the cells by the perchloric acid extraction method, as described previously. ${ }^{13,15}$

\section{Molecular modeling of $\mathrm{AT}_{1}$ receptor-ARBs}

The amino-acid sequences of human $\mathrm{AT}_{1}$ receptor, bovine rhodopsin (PDBID:1U19), human $\beta_{2}$ adrenergic receptor (PDBID:2RH1) and human $\mathrm{A}_{2 \mathrm{~A}}$ adenosine receptor (PDBID:3 EML) were added to a multiple sequence alignment comprising 68 sequences in the purine receptor and to the peptide clusters reported by Costanzi et al. ${ }^{16}$ We carried out multiple sequence alignment including these sequences using the MOE software (version 2010.10, Chemical Computing Group, Montreal, Quebec, Canada). The BLOSUM62 matrix ${ }^{17}$ was applied, with a gap start penalty of 5 and a gap extend penalty of 0.2 .

Homology models were built with the program MODELLER 9v7 (Accelrys, San Diego, CA, USA) using rhodopsin, $\beta_{2}$ adrenergic receptor or $A_{2 A}$ adenosine receptor as a template protein. Three thousand homology models were generated from each template protein. The extracellular 2 (EL2) loop was built completely de novo, without any alignment between the sequences of $\mathrm{AT}_{1}$ receptor and the template protein, but the disulfide bridge of the conserved Cys residues on the transmembrane (TM) 3 helix and EL2 loop was enforced.

Azilsartan was docked into the homology models using the SP algorithm within Schrödinger Glide 5.6 software (Schrödinger, New York, NY, USA). The protein preparation and grid-generation processes were performed automatically using the XGlide python script (Schrödinger). Amino-acid residues $\mathrm{Gln}^{257}$, Lys ${ }^{199}$ and $\mathrm{Asn}^{295}$ were used to define the cavity of the grid. $\mathrm{Gln}^{257}$ and Lys ${ }^{199}$ were identified as important residues for the interaction between azilsartan and $\mathrm{AT}_{1}$ receptor by mutagenesis experiments as described in the Results section, and $\mathrm{Asn}^{295}$ was used to dock the ligand inside the membrane protein. No constraints were added to grid generation to ensure that subsequent dockings were not biased in any way. One pose per protein in the docking process was stored for analysis. Model selection was performed using the protein ligand interaction fingerprints module within MOE. We chose five models with ligands that showed both hydrogen bonding with $\mathrm{Gln}^{257}$ and ionic interaction with Lys ${ }^{199}$. To refine these models, the induced fit docking protocol within Schrödinger software was applied, and generated 100 refined models from each docking model. Among these models, the model that had the best induced fit docking score ${ }^{18}$ was selected. For comparison with the binding model of azilsartan, candesartan was docked into the structure of the best model of the azilsartan/ $\mathrm{AT}_{1}$ receptor using the Glide SP algorithm (Schrödinger). Finally, the docking model of candesartan/ $\mathrm{AT}_{1}$ receptor was minimized to only amino-acid residues that are within $5 \AA$ around the ligand using MacroModel 9.8 within Schrödinger software.

\section{Statistical analysis}

The results are expressed as the mean \pm s.d. of four or more independent determinations. Significant differences in measured values were evaluated with an analysis of variance using Fisher's $t$-test and unpaired Student's $t$-test. Statistical significance was set at $<0.05$.

\section{RESULTS}

Binding affinities of azilsartan, azilsartan-7H and candesartan to $\mathrm{WT}$ and mutant $\mathrm{AT}_{1}$ receptors

The $K_{\mathrm{d}}$ of azilsartan for the WT $\mathrm{AT}_{1}$ receptor was comparable to that of candesartan. Next, we selected candidate amino acids in the $\mathrm{AT}_{1}$ receptor $\left(\mathrm{Ser}^{105}, \mathrm{Ser}^{109}, \mathrm{Tyr}^{113}, \mathrm{Val}^{116}, \mathrm{Ph}^{182}, \mathrm{Tyr}^{184}, \mathrm{Lys}^{199}, \mathrm{Phe}^{208}\right.$, $\operatorname{Trp}^{253}$, $\mathrm{His}^{256}, \mathrm{Gln}^{257}$, $\mathrm{Thr}^{287}, \mathrm{Tyr}^{292}$ and $\mathrm{Asn}^{295}$, Supplementary Figure 2) for consideration as specific binding sites of azilsartan based on the molecular models of the $\mathrm{AT}_{1}$ receptor complex described in previous reports. ${ }^{12,13,19,20}$ To determine the specific site that binds to azilsartan, we examined the binding affinities of azilsartan, azilsartan-7H and candesartan, the chemical structures of which are shown in Figure 1, to $\mathrm{AT}_{1}$ receptors that were mutated at the candidate amino acids mentioned above. The results are shown in Table 1. The affinities of $\left[\mathrm{Sar}^{1}, \mathrm{Il}^{8}\right]$ Ang II were almost the same for some mutants and were decreased for other mutants, but not to $<1 / 10$ the affinity for the $\mathrm{WT}_{\mathrm{AT}} \mathrm{T}_{1}$ receptor. The affinity of azilsartan for the Y113A, K199A, Q257A and N295A mutants was reduced by $>10$-fold compared with the $\mathrm{WT}_{\mathrm{AT}} \mathrm{1}_{1}$ receptor, suggesting that $\mathrm{Tyr}^{113}$, $\mathrm{Lys}^{199}, \mathrm{Gln}^{257}$ and $\mathrm{Asn}^{295}$ in the $\mathrm{AT}_{1}$ receptor are involved in binding 

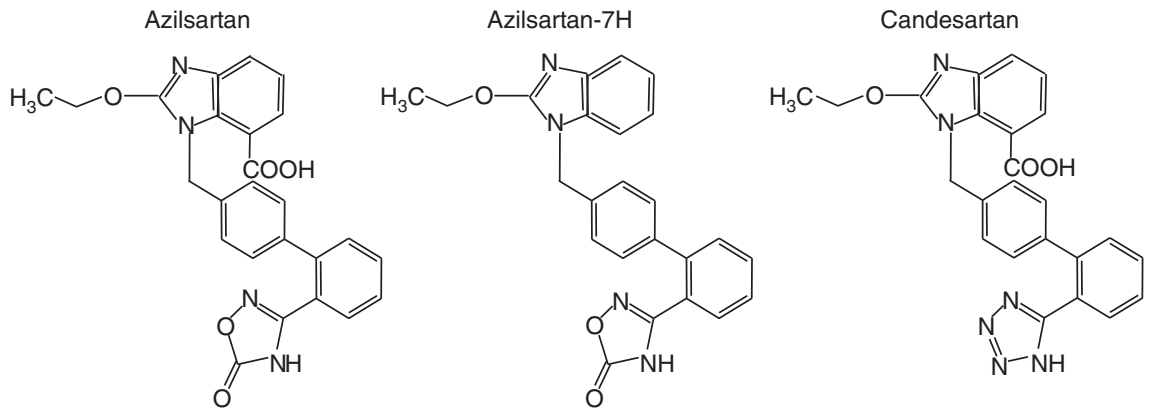

Figure 1 Chemical structures of ARBs.

Table 1 Binding affinities $\left(K_{d}\right)$ of Ang II and ARBs to AT 1 WT and mutants receptors

\begin{tabular}{|c|c|c|c|c|}
\hline & $\begin{array}{c}{\left[\mathrm{Sar}^{1}, \| \mathrm{e}^{8}\right]} \\
\text { Ang } / /\end{array}$ & & & \\
\hline WT & $0.7 \pm 0.1(1.0)$ & $2.6 \pm 0.5(1.0)$ & $3.1 \pm 0.2(1.0)$ & $49 \pm 9(1.0)$ \\
\hline S105A & $0.5 \pm 0.3(0.7)$ & $5.4 \pm 2.0(2.1)$ & $12 \pm 3(3.9)$ & $33 \pm 5(0.7)$ \\
\hline S109A & $1.4 \pm 0.1(2.0)$ & $5.6 \pm 0.7(2.2)$ & $11 \pm 3(3.5)$ & $29 \pm 9(0.6)$ \\
\hline $\mathrm{N} 111 \mathrm{G}$ & $0.8 \pm 0.5(1.0)$ & $15 \pm 5(5.8)$ & $48 \pm 6(15)$ & $2362 \pm 756$ (48) \\
\hline $\mathrm{N} 111 \mathrm{G} /$ & $1.3 \pm 0.9(1.9)$ & $241 \pm 41(93)$ & $754 \pm 54(243)$ & $13654 \pm 667$ (279) \\
\hline K199Q & & & & \\
\hline & $1.0 \pm 0.2(1.4)$ & $64 \pm 18(25)$ & $71 \pm 27(15)$ & $4477 \pm 437(91)$ \\
\hline Y113A & $1.0 \pm 0.4(1.4)$ & $173 \pm 45(67)$ & $232 \pm 96(75)$ & $14794 \pm 482(302)$ \\
\hline Y113F & $1.8 \pm 1.0(2.6)$ & $7.9 \pm 3.6$ & $14 \pm 0.3(4.5)$ & $523 \pm 128(11)$ \\
\hline V116A & $1.0 \pm 0.4(1.4)$ & $7.4 \pm 2.7(2.8)$ & $9.6 \pm 0.8(3.1)$ & $76 \pm 23$ (1.6) \\
\hline $\mathrm{F} 182 \mathrm{~A}$ & $0.6 \pm 0.2(0.9)$ & $3.2 \pm 0.5(1.2)$ & $7.1 \pm 0.9(2.3)$ & $23 \pm 5(0.5)$ \\
\hline Y184A & $1.0 \pm 0.4(1.4)$ & $2.0 \pm 0.7(1.2)$ & $4.8 \pm 1.3(2.3)$ & $24 \pm 9(0.5)$ \\
\hline K199A & $3.3 \pm 0.5(4.7)$ & $53 \pm 5(20)$ & $103 \pm 7$ (33) & $2059 \pm 344(42)$ \\
\hline F208A & $0.7 \pm 0.3(1.0)$ & $3.6 \pm 1.2(1.4)$ & $7.5 \pm 1.9(2.4)$ & $30 \pm 12(0.6)$ \\
\hline W253A & $2.2 \pm 0.8(3.1)$ & $13 \pm 2(5.0)$ & $25 \pm 4(8.1)$ & $40 \pm 7(0.8)$ \\
\hline $\mathrm{H} 256 \mathrm{~A}$ & $0.8 \pm 0.3(1.1)$ & $4.3 \pm 1.6(1.7)$ & $5.6 \pm 2.4(1.8)$ & $54 \pm 11(1.1)$ \\
\hline Q257A & $2.5 \pm 0.2(3.6)$ & $37 \pm 8(14)$ & $309 \pm 22(100)$ & $5028 \pm 1216$ \\
\hline T287A & $1.3 \pm 0.2(1.9)$ & $15 \pm 5(5.8)$ & $30 \pm 7(9.7)$ & $741 \pm 35$ (15) \\
\hline Y292A & $1.7 \pm 0.8(2.4)$ & $4.6 \pm 1.4(1.8)$ & $18 \pm 5(5.8)$ & $22 \pm 4(0.4)$ \\
\hline N295A & $6.4 \pm 2.1(9.3)$ & $99 \pm 16$ (38) & $212 \pm 97(68)$ & $2291 \pm 861$ \\
\hline
\end{tabular}

Abbreviations: Ang II, angiotensin II; ARB, $\mathrm{AT}_{1}$ receptor blocker; AT1, angiotensin II type 1; WT, wild type.

Numbers in parentheses show ratio of $K_{d}$ (mutant) $/ K_{d}(\mathrm{WT})$.

to azilsartan. Interestingly, azilsartan (100-fold reduction in binding affinity to $\mathrm{WT} \mathrm{AT}_{1}$ receptor) exhibited about a sevenfold greater reduction in binding affinity to the Q257A receptor compared with candesartan (14-fold reduction), indicating that the oxadiazole of azilsartan probably binds to $\mathrm{Gln}^{257}$ of the $\mathrm{AT}_{1}$ receptor. In addition, the affinity of azilsartan-7H $(49 \mathrm{~nm})$ for the $\mathrm{AT}_{1}$-WT receptor was about 16-fold less than that of azilsartan $(3.1 \mathrm{nM})$, and the affinity of azilsartan was comparable to that of candesartan, suggesting that the carboxyl moiety of azilsartan is also important for binding to the $\mathrm{AT}_{1}$-WT receptor.

Binding and inverse agonism properties of azilsartan, azilsartan7H and candesartan

Next, we examined whether azilsartan, azilsartan-7H and candesartan retained the ability to bind to N111G mutant receptor (Table 1). As we previously reported that the $\mathrm{AT}_{1}-\mathrm{N} 111 \mathrm{G}$ receptor had high basal activity in the absence of Ang $\mathrm{II}^{21}$ and could be used to determine the inverse agonism of azilsartan, we also analyzed the binding affinities of these ARBs in the mutant receptor. The affinity of azilsartan-7H (5754 nM) for the $\mathrm{AT}_{1}-\mathrm{N} 111 \mathrm{G}$ receptor was 113 -fold less than that of azilsartan $(51 \mathrm{~nm})$. These data suggest that the carboxyl moiety of azilsartan is important for binding to the N111G receptor.

We analyzed whether azilsartan induced inverse agonism toward IP production on WT, N111G, N111G/K199Q and N111G/Q257A receptors (Figure 2). Azilsartan significantly suppressed the basal activity of WT receptor. However, the basal activity of the WT AT receptor is too low to evaluate the differences in inverse agonistic activity among ARBs. To confirm these differences, we used N111G receptor. Although both azilsartan and candesartan significantly suppressed the basal activities of the mutant receptor, azilsartan showed significantly stronger inverse agonism than candesartan. Modification of the carboxyl moiety of azilsartan gave azilsartan$7 \mathrm{H}$, which did not show inverse agonism and instead showed neutral antagonism. In addition, azilsartan did not induce inverse agonism in the N111G/K199Q and N111G/Q257A receptors. Thus, the results suggested that the insertion of oxadiazole in the tetrazole ring in azilsartan induced stronger inverse agonism and the position of $\mathrm{Gln}^{257}$ in addition to $\mathrm{Lys}^{199}$ in the $\mathrm{AT}_{1}$ receptor may have a role in the inverse agonism of azilsartan.

\section{Molecular model of the interaction between azilsartan and the $\mathrm{AT}_{1}$ receptor}

A molecular model was constructed based on the three main interactions between the $\mathrm{AT}_{1}$ receptor and azilsartan that were suggested from the mutation experiments (Figure 3). Gln ${ }^{257}$ binds to the oxadiazole ring by hydrogen bonding, with a bond distance of $2.6 \AA$. This distance was shorter than that between $\operatorname{Gln}^{257}$ and the tetrazol ring of candesartan $(3.3 \AA)$, indicating that the interaction may form stronger hydrogen bonding. Tyr ${ }^{113}$ binds to the biphenyl group of azilsartan by Van der Waals interaction, as the mutagenesis data indicated that the phenyl group of $\mathrm{Tyr}^{113}$ would be more important for interaction with azilsartan than the hydroxyl group, and the atomic distance was $3.4 \AA$. This distance was shorter than that between $\operatorname{Tyr}^{113}$ and the biphenyl group of candesartan $(4.0 \AA)$. In addition, Lys ${ }^{199}$ was a candidate for binding to the carboxyl group of azilsartan, and the bond distances were 2.6 and $3.2 \AA$.

\section{DISCUSSION}

This study demonstrated that azilsartan induces stronger inverse agonism than candesartan and this ability of azilsartan may be associated with its unique moiety, a 5-oxo-1,2,4-oxadiazole, in place of a tetrazole ring. The oxadiazole ring in azilsartan is not found in any other clinically approved ARBs, as most ARBs, including candesartan, have a biphenylmethyl moiety with an acidic group 


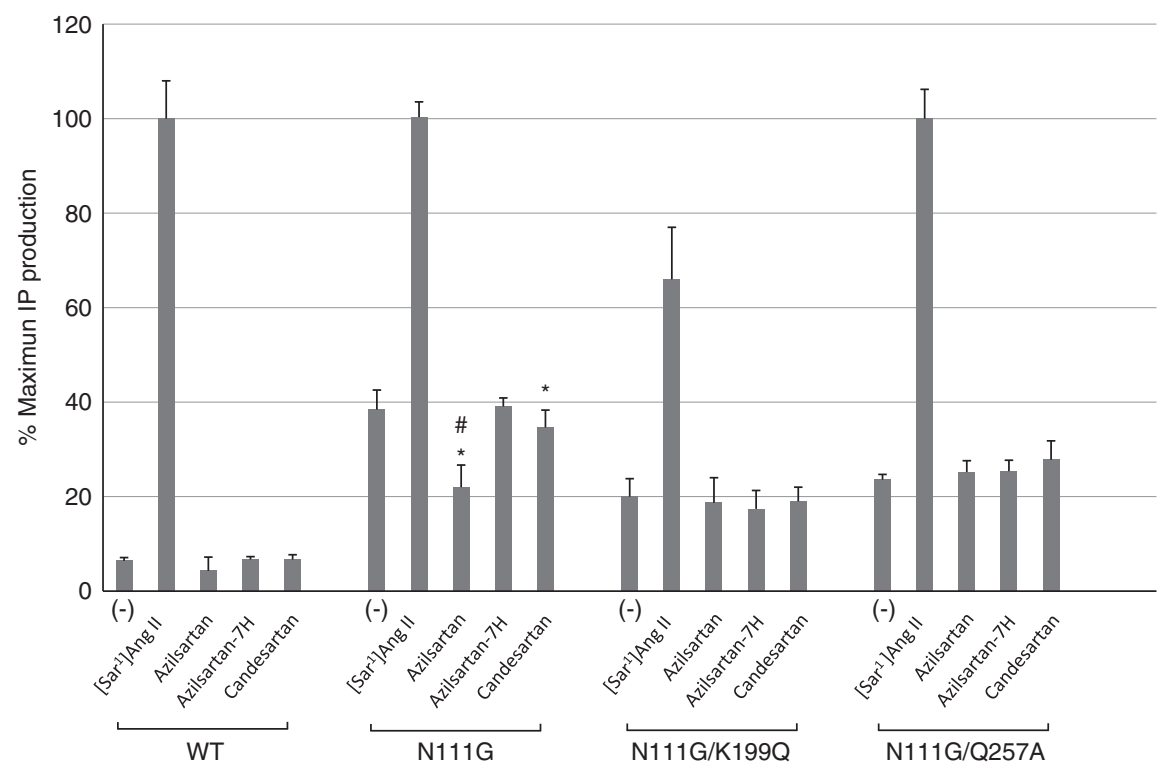

Figure 2 Percent of maximum IP production with or without $1 \mu \mathrm{m}$ of the ARBs or $0.1 \mu \mathrm{m}$ [Sar ${ }^{1}$ ]Ang II in COS1 cells transiently expressing the WT, N111G, $\mathrm{N} 111 \mathrm{G} / \mathrm{K} 199 \mathrm{Q}$ or N111G/Q257A AT 1 receptor. [Sar ${ }^{1}$ ]Ang II or ARB was added to the medium for 10 min. Percentage (\%) maximum IP production indicates [Sar ${ }^{1}$ ]Ang II-induced IP production (1920 c.p.m.) in WT AT 1 receptor-transfected cells (100\%) after adjusting for basal IP production (130 c.p.m.) without treatment in mock-treated cells $(0 \%)$. ${ }^{*} P<0.05$ vs. no treatment. ${ }^{\#} P<0.05$ vs. candesartan.

a

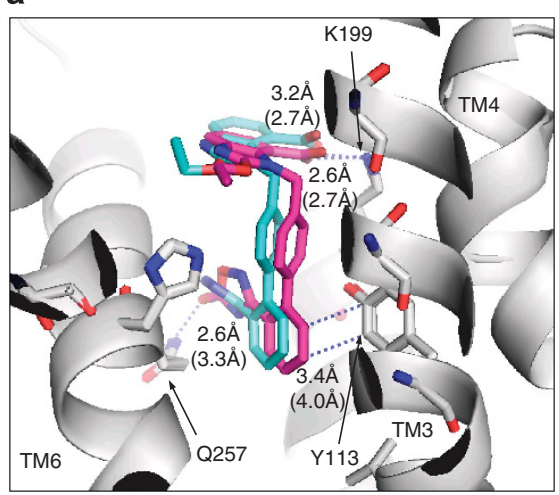

b

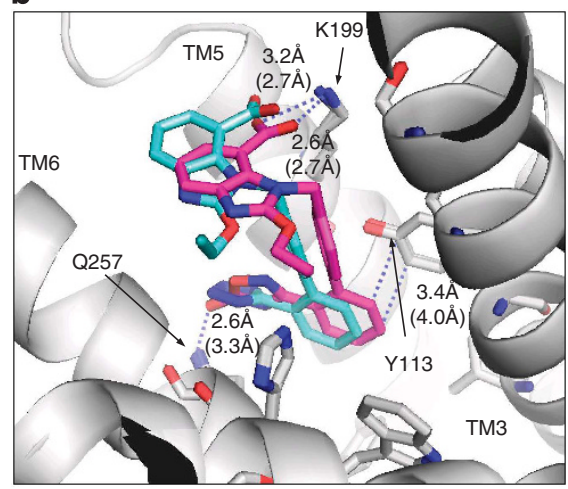

Figure 3 Molecular model of the interaction between azilsartan (magenta) or candesartan (blue) and the $\mathrm{AT}_{1}$ receptor. (a) and (b) are side and top views, respectively. The $\mathrm{AT}_{1}$ receptor is shown as a ribbon. Numbers (in parentheses) show the distances between azilsartan (candesartan) and the sites of the $\mathrm{AT}_{1}$ receptor.

(either a tetrazole or carboxylic acid). A molecular model suggested that $\mathrm{Gln}^{257}$ binds to the oxadiazole ring by hydrogen bonding, and the bond distance was shorter than that between $\operatorname{Gln}^{257}$ and the tetrazol ring of candesartan.

Fabia et al. ${ }^{22}$ studied 36 reports in which BP was measured using ambulatory BP monitoring. The antihypertensive activities of ARBs differed, and the magnitude of the reduction in BP did not essentially depend on the initial BP values or on the dose used. In addition, azilsartan showed a more potent $24 \mathrm{~h}$ sustained antihypertensive effect than candesartan. ${ }^{5}$ Thus, not all ARBs may have the same antihypertensive effects. Although spontaneous mutations have not been reported for the $\mathrm{AT}_{1}$ receptor, we reported that the $\mathrm{WT} \mathrm{AT}_{1}$ receptor shows slight but significant constitutive activity with regard to the accumulation of IP, which is a cell signaling molecule that has a role in vasoconstriction. ${ }^{12}$ An inverse agonist can inhibit the constitutive activity of $\mathrm{AT}_{1}$ receptor and may induce a stronger
BP-lowering effect than a neutral antagonist toward IP production. Azilsartan showed stronger inverse agonism than candesartan, and this could help explain why azilsartan showed a stronger antihypertensive effect than candesartan. Although $\mathrm{WT} \mathrm{AT}_{1}$ receptor shows only slight constitutive activity, Morisset et al. ${ }^{23}$ clearly showed that inverse agonists are useful in a therapeutic strategy even if nonmutated receptors are expressed at normal levels in G-proteincoupled receptors, $\mathrm{H}_{3}$ receptor. Although we understand that the inverse agonism of azilsartan based on an experimental study may not necessarily directly influence the clinical outcome, we believe that it is reasonable to consider this possibility because the concentration of azilsartan $(1 \mu \mathrm{M})$ in the experimental study was comparable to the azilsartan concentration in human plasma after the administration of azilsartan in a dose of $20-40 \mathrm{mg}$.

Moreover, there have been several reports that may explain why azilsartan lowers BP more than other ARBs. ${ }^{11,24,25}$ First, Ojima et al. ${ }^{11}$ 
reported that azilsartan inhibited the accumulation of Ang II-induced IP in a cell-based assay, and this effect was resistant to washout. Although olmesartan and valsartan inhibited Ang II-induced IP accumulation, the activities of these compounds were markedly reduced after washout. Time-course studies of the abilities of different $\mathrm{ARBs}$ to persistently block Ang II binding to $\mathrm{AT}_{1}$ receptors after drug washout for $240 \mathrm{~min}$ have also indicated that azilsartan dissociates from $\mathrm{AT}_{1}$ receptors more slowly than other $\mathrm{ARBs}$, including olmesartan, telmisartan and valsartan. Second, azilsartan medoxomil inhibited Ang II-induced pressor responses in rats, and its inhibitory effects lasted $24 \mathrm{~h}$ after oral administration, whereas the inhibitory effects of olmesartan medoxomil disappeared within $24 \mathrm{~h} .^{24}$ Third, azilsartan blocked Ang II-induced activation of mitogenactivated protein kinase in vascular smooth muscle cells $4-8 \mathrm{~h}$ after washout of the drug from the incubation media. ${ }^{25}$

Azilsartan has a unique moiety, a 5-oxo-1,2,4-oxadiazole, in place of a tetrazole ring. On the basis of molecular modeling, $\operatorname{Gln}^{257}$ binds to the oxadiazole ring by hydrogen bonding that is stronger than the hydrogen bonding of $\mathrm{Gln}^{257}$ to the tetrazol ring of candesartan, as the bond distance in azilsartan was shorter than that in candesartan. In addition, Lys ${ }^{199}$ and $\mathrm{Tyr}^{113}$ may bind to the carboxyl group and biphenyl group of azilsartan, respectively. These three interactions are critical for the tight binding of azilsartan to $\mathrm{AT}_{1}$ receptor. When the receptor is viewed from the top, azilsartan seems to form the letter ' $S$ ' when it binds (Supplementary Figure 3). The oxadiazole in azilsartan, which represents a small difference in the chemical structure compared with candesartan, induced stronger inverse agonism, and the position of $\mathrm{Gln}^{257}$ (TM6) in addition to Lys ${ }^{199}$ (TM5) in the $\mathrm{AT}_{1}$ receptor may have a role in the inverse agonistic activity of azilsartan. We previously reported that olmesartan and valsartan showed inverse agonism toward IP production. ${ }^{12,13}$ Cooperative interactions between the hydroxyl group and $\mathrm{Tyr}^{113}$ (TM3) and between the carboxyl group and $\mathrm{His}^{256}$ (TM6) are crucial for the inverse agonist activity of olmesartan, ${ }^{12}$ whereas the most critical interaction for the inverse agonism of valsartan involves Lys ${ }^{199}$ of $\mathrm{AT}_{1}$ receptor. The molecular basis of the inverse agonism of azilsartan was found to be distinct from that of olmesartan and valsartan.

One of the critical features of $\mathrm{ARBs}$ is selectivity for the $\mathrm{AT}_{1}$ receptor over the $\mathrm{AT}_{2}$ receptor. The high selectivity is expected to lead to additional $\mathrm{AT}_{2}$ stimulation because local free Ang II levels will rise after $\mathrm{ARB}$ treatment. ${ }^{26} \mathrm{AT}_{2}$ receptor stimulation induces vasodilation through bradykinin and nitric oxide release, cell growth inhibition and natriuresis. ${ }^{27}$ The greatest difference in affinities for $\mathrm{AT}_{1}$ and $\mathrm{AT}_{2}$ has been reported for valsartan, at 30000 times greater affinity for the $\mathrm{AT}_{1}$ receptor than for the $\mathrm{AT}_{2}$ receptor. ${ }^{28}$ In the case of azilsartan, the selectivity was about 39000 times higher for the $\mathrm{AT}_{1}$ receptor than for the $\mathrm{AT}_{2}$ receptor according to our experiment (the $K_{\mathrm{d}}$ of azilsartan for the $\mathrm{AT}_{1}$ and $\mathrm{AT}_{2}$ receptors was $3.1 \mathrm{~nm}$ and $121 \mu \mathrm{M}$, respectively; Miura $\mathrm{S}$ et al. our unpublished data), indicating that azilsartan may have a beneficial effect in this respect.

Although ARBs are generally well tolerated, the development of new ARBs is important because the currently available ARBs cannot achieve optimal BP levels, and many patients continue to suffer from cardiovascular events and metabolic disturbances despite being treated with an ARB. ${ }^{29,30}$ In this respect, as azilsartan bound tightly to and dissociated slowly from $\mathrm{AT}_{1}$ receptors compared with other ARBs, azilsartan is expected to be a desirable ARB because it not only shows superior BP control compared with other ARBs but also improves insulin resistance in animal models. ${ }^{24,25}$

In conclusion, this study demonstrated that azilsartan induces stronger inverse agonism than candesartan, and this ability of azilsartan may be associated with its unique moiety, a 5-oxo-1,2,4oxadiazole, in place of a tetrazole ring. The oxadiazole ring in azilsartan is not found in any other clinically approved ARB. A mutagenesis study and molecular modeling revealed that Gln ${ }^{257}$ in the $\mathrm{AT}_{1}$ receptor binds to the oxadiazole ring of azilsartan by stronger hydrogen bonding than with candesartan. This interaction may tightly bind azilsartan to the $\mathrm{AT}_{1}$ receptor and could be important for inducing inverse agonism.

\section{CONFLICT OF INTEREST}

SM has received lecture honoraria from Takeda Pharm Co. Ltd. AO is a full-time employee of Takeda Pharm Co. Ltd.

\section{ACKNOWLEDGEMENTS}

We thank S Tomita for providing excellent technical assistance. This work was supported in part by a Grant-in-Aid for Scientific Research (21591065) from the Ministry of Education, Culture, Sports, Science and Technology, Japan.

1 De Gasparo M, Catt KJ, Inagami T, Wright JW, Unger T. International union of pharmacology. XXIII. The angiotensin II receptors. Pharmacol Rev 2000; 52 415-472.

2 White WB, Weber MA, Sica D, Bakris GL, Perez A, Cao C, Kupfer S. Effects of the angiotensin receptor blocker azilsartan medoxomil versus olmesartan and valsartan on ambulatory and clinic blood pressure in patients with stages 1 and 2 hypertension. Hypertension 2011; 57: 413-420.

3 Bakris GL, Sica D, Weber M, White WB, Roberts A, Perez A, Cao C, Kupfer S. The comparative effects of azilsartan medoxomil and olmesartan on ambulatory and clinic blood pressure. J Clin Hypertens 2011; 13: 81-88.

4 Sica D, White WB, Weber MA, Bakris GL, Perez A, Cao C, Handley A, Kupfer S. Comparison of the novel angiotensin II receptor blocker azilsartan medoxomil vs. valsartan by ambulatory blood pressure monitoring. J Clin Hypertens $2011 ; 13$ $467-472$

5 Rakugi H, Enya K, Sugiura K, Ikeda Y. Comparison of the efficacy and safety of azilsartan with that of candesartan cilexetil in Japanese patients with grade I-II essential hypertension: a randomized, double-blind clinical study. Hypertens Res 2012; 35: 552-558.

6 Kohara Y, Kubo K, Imamiya E, Wada T, Inada Y, Naka T. Synthesis and angiotensin II receptor antagonistic activities of benzimidazole derivatives bearing acidic heterocycles as novel tetrazole bioisosteres. J Med Chem 1996; 39: 5228-5235.

7 Miura S, Karnik SS, Saku K. Review: angiotensin II type 1 receptor blockers: class effects versus molecular effects. Renin Angiotensin Aldosterone Syst 2011; 12: $1-7$.

8 Fujino M, Miura S, Kiya Y, Tominaga Y, Matsuo Y, Karnik SS, Saku K. A smal difference in the molecular structure of angiotensin II receptor blockers induces ATI receptor-dependent and -independent beneficial effects. Hypertens Res 2010; 33: 1044-1052.

9 Soudijn W, van Wijngaarden I, Ijzerman AP. Structure-activity relationships of inverse gonists for G-protein-coupled receptors. Med Res Rev 2005; 25: 398-426.

10 Rossier O, Abuin L, Fanelli F, Leonardi A, Cotecchia S. Inverse agonism and neutra antagonism at alpha(1a)- and alpha(1b)-adrenergic receptor subtypes. Mol Pharmacol 1999; 56: 858-866

11 Ojima M, Igata H, Tanaka M, Sakamoto H, Kuroita T, Kohara Y, Kubo K, Fuse H, Imura $\mathrm{Y}$, Kusumoto K, Nagaya H. In vitro antagonistic properties of a new angiotensin type 1 receptor blocker, azilsartan, in receptor binding and function studies. J Pharmacol Exp Ther 2011; 336: 801-808.

12 Miura S, Kiya Y, Kanazawa T, Imaizumi S, Fujino M, Matsuo Y, Karnik SS, Saku K. Differential bonding interactions of inverse agonists of angiotensin II type 1 receptor in stabilizing the inactive state. Mol Endocrinol 2008; 22: 139-146.

13 Miura S, Fujino M, Hanzawa H, Kiya Y, Imaizumi S, Matsuo Y, Tomita S, Uehara Y, Karnik SS, Yanagisawa H, Koike H, Komuro I, Saku K. Molecular mechanism underlying inverse agonist of angiotensin II type 1 receptor. J Biol Chem 2006; 281: 19288-19295.

14 Soudijn W, van Wijngaarden I, Ijzerman AP. Structure-activity relationships of inverse agonists for G-protein-coupled receptors. Med Re Rev 2005; 25: 398-426.

15 Miura S, Feng YH, Husain A, Karnik SS. Role of aromaticity of agonist switches of angiotensin II in the activation of the AT1 receptor. J Biol Chem 1999; 274 7103-7110.

16 Costanzi S, Mamedova L, Gao ZG, Jacobson KA. Architecture of P2Y nucleotide receptors: Structural comparison based on sequence analysis, mutagenesis, and homology modeling. J Med Chem 2004; 47: 5393-5404.

17 Henikoff S, Henikoff JG. Amino acid substitution matrices from protein blocks. Proc Natl Acad Sci USA 1992; 89: 10915-10909.

18 Sherman W, Day T, Jacobson MP, Friesner RA, Farid R. Novel procedure for modeling ligand/receptor induced fit effects. J Med Chem 2006; 49: 534-554. 
19 Noda K, Saad Y, Kinoshita A, Boyle TP, Graham RM, Husain A, Karnik SS. Tetrazole and carboxylate groups of angiotensin receptor antagonists bind to the same subsite by different mechanisms. J Biol Chem 1995; 270: 2284-2289.

20 Takezako T, Gogonea C, Saad Y, Noda K, Karnik SS. 'Network leaning' as a mechanism of insurmountable antagonism of the angiotensin II type 1 receptor by non-peptide antagonists. J Biol Chem 2004; 279: 15248-15257.

21 Feng YH, Miura S, Husain A, Karnik SS. Mechanism of constitutive activation of the AT1 receptor: influence of the size of the agonist switch binding residue Asn(111). Biochemistry 1998; 37: 15791-15798.

22 Fabia MJ, Abdilla N, Oltra R, Fernandez C, Redon J. Antihypertensive activity of angiotensin II $\mathrm{AT}_{1}$ receptor antagonists: a systematic review of studies with $24 \mathrm{~h}$ ambulatory blood pressure monitoring. J Hypertens 2007; 25: 1327-1336.

23 Morisset S, Rouleau A, Ligneau X, Gbahou F, Tardivel-Lacombe J, Stark H, Schunack W, Ganellin CR, Schwartz JC, Arrang JM. High constitutive activity of native H3 receptors regulates histamine neurons in brain. Nature 2000; 408: 860-864.

24 Kusumoto K, Igata H, Ojima M, Tsuboi A, Imanishi M, Yamaguchi F, Sakamoto H, Kuroita T, Kawaguchi N, Nishigaki N, Nagaya H. Antihypertensive insulin-sensitising and renoprotective effects of a novel, potent and long-acting angiotensin II type 1 receptor blocker, azilsartan medoxomil, in rat and dog models. Eur J Pharmacol 2011; 339: 84-93.

25 Kajiya T, Ho C, Wang J, Vilardi R, Kurtz TW. Molecular and cellular effects of azilsartan: a new generation angiotensin II receptor blocker. J Hypertens 2011; 29: 2476-2483.

26 Siragy HM, de Gasparo M, Carey RM. Angiotensin type 2 receptor mediates valsartaninduced hypotension in conscious rats. Hypertension 2000; 35: 1074-1077.

27 Siragy HM. Angiotensin receptor blockers: how important is selectivity? Am J Hypertens 2002; 15: 1006-1014.

28 de Gasparo M, Whitebread S. Binding of valsartan to mammalian angiotensin AT1 receptors. Regul Pept 1995; 59: 303-311.

29 Kurtz TW, Klein U. Next generation multifunctional angiotensin receptor blockers. Hypertens Res 2009; 32: 826-834.

30 Verdecchia P, Angeli F, Mazzotta G, Ambrosio G, Reboldi G. Angiotensin receptor blockers in hypertension. New insights from Japan. Hypertens Res 2010; 33: 394-397.

Supplementary Information accompanies the paper on Hypertension Research website (http://www.nature.com/hr) 